UDC 539.17

\author{
*Burkova N.A., Tkachenko A.S. \\ Faculty of physics and technology, al-Farabi Kazakh National University, \\ Almaty, Kazakhstan \\ "e-mail: natali.burkova@gmail.com
}

\title{
Algebraic basics for high spin elastic scattering
}

\begin{abstract}
The analytics was implemented for the scattering processes with channel spin structure 1/2, 3/2 and $5 / 2$. For the first time the parameterization of differential cross sections by orbital momentums $\ell=0$, $1,2, \ldots$ for the quartet and quintet spin channels has been performed. The presentation is given in a universe form and may be applied for the phase shift analyses for the scattering of nuclei or elementary particles. Same amplitudes are used in the construction of the polarization characteristics as vector and tensor polarization. The following isotopes are in field of interests: ${ }^{7} \mathrm{~B}\left(3 / 2^{-}\right),{ }^{9} \mathrm{~B}\left(3 / 2^{-}\right),{ }^{11} \mathrm{~B}\left(3 / 2^{-}\right)$, ${ }^{12} \mathrm{~B}\left(1^{+}\right),{ }^{13} \mathrm{~B}\left(3 / 2^{-}\right),{ }^{5} \operatorname{Li}\left(3 / 2^{-}\right),{ }^{6} \operatorname{Li}\left(1^{+}\right),{ }^{7} \operatorname{Li}\left(3 / 2^{-}\right),{ }^{9} \operatorname{Li}\left(3 / 2^{-}\right),{ }^{10} \operatorname{Li}\left(1^{-}\right),{ }^{9} \mathrm{C}\left(3 / 2^{-}\right),{ }^{11} \mathrm{C}\left(3 / 2^{-}\right)$and protons, neutrons, deuterons as impact particles.
\end{abstract}

Key words: elastic scattering, spin channel, probability amplitude, cross section, partial wave.

\section{Introduction}

Of particular interest to the two-particle photodisintegration and radiative capture reactions $A+\gamma \rightleftarrows a+b$ is due to the fact that these processes are directly related to the most advanced research in nuclear astrophysics (thermodynamics of the Universe [1], nucleosynthesis of chemical elements in the Sun and stars, the Big Bang theory [2]), as well as the physics of nuclear reactors and nuclear fusion.

Currently, with implementation of the novel method of inverse Compton scattering (ICPB) for the generating a quasi-monochromatic beams of photons experimental capabilities have reached a new level and is continuously expanding.

It should also be noted, that in $A+\gamma \rightleftarrows a+b$ reactions both the discrete spectrum and continuous spectrum are involved. Thus, for the sequential description of $a+b$ scattering channel necessary to have the interaction potential. The parameters for such phenomenological potentials might be reconstructed as fitting the data on the cross sections of elastic scattering.

By now the scattering theory of both classical and quantum, is one of the most developed fields of theoretical physics. Mathematical formalism for the non-relativistic collision theory has been developed well and is represented in numerous original papers and reviews [3-8], university textbooks [9-10] and monographs [11-12].
However, for the practical application of this theory there are real difficulties connected with the fact that characteristics such as, for example, the density matrix and scattering matrix contain a defined set of some parameters, the number of which greatly increases when account is taken of the spin structure of the particles involved in the processes. In this context, the relevance of a particular task on the construction of the scattering matrix and the calculation of its elements is associated with the minimization of the number of parameters used. This requires an analysis of all the known symmetry properties of the system under study

\section{Parameterization of differential cross sections}

For practical calculations of the differential cross sections of scattering processes in a wide range of energies the elastic channels is enough to take into account, that is, with good accuracy, to consider only the spin-orbit potential $V_{l s}$, neglecting the spin-spin interaction. In this case, the matrix $M$ is written explicitly:

$$
\begin{gathered}
M_{s v v^{\prime}}(\theta)=\sqrt{\pi} k^{-1}\left[-C(\theta) \delta_{v v^{\prime}}+\right. \\
+i \sum_{J \ell}(2 \ell+1)^{1 / 2} C_{s v \ell 0}^{J v} C_{s v^{\prime} \ell m}^{J v} . \\
\left.\cdot \exp \left(2 i \sigma_{\ell}\right)\left(1-U_{s \ell}^{J^{\pi}}\right) Y_{\ell}^{m}(\theta, 0)\right],
\end{gathered}
$$


where $S$-spin channel, $v$ and $v^{\prime}$ - corresponding spin projections on $\mathrm{Z}$ axis, $C(\theta)$ - Coulomb scattering amplitude, $\ell$ and $\ell^{\prime}-$ orbital quantum numbers in initial and final channels, $C_{j_{1} m_{1} j_{2} m_{2}}^{j m}-$ Clebsh-Gordan coefficients [13], $\sigma_{\ell}-$ coulomb phase shifts, $U_{s^{\prime} \ell}^{J^{\pi} s \ell}-$ scattering matrix, $Y_{\ell^{\prime}}^{m^{\prime}}(\theta, 0)-$ angular functions. Note that with increasing values of the spin channel increases its contribution to the total differential cross section.

Here we oriented on the future research of the elastic scattering processes of particles with spin numbers 1 and $3 / 2$. As a participants the following isotopes are in field of interests: ${ }^{7} \mathrm{~B}\left(3 / 2^{-}\right)$, ${ }^{9} \mathrm{~B}\left(3 / 2^{-}\right), \quad{ }^{11} \mathrm{~B}\left(3 / 2^{-}\right), \quad{ }^{12} \mathrm{~B}\left(1^{+}\right), \quad{ }^{13} \mathrm{~B}\left(3 / 2^{-}\right)$, ${ }^{5} \mathrm{Li}\left(3 / 2^{-}\right), \quad{ }^{6} \mathrm{Li}\left(1^{+}\right), \quad{ }^{7} \mathrm{Li}\left(3 / 2^{-}\right), \quad{ }^{9} \mathrm{Li}\left(3 / 2^{-}\right)$, ${ }^{10} \mathrm{Li}\left(1^{-}\right),{ }^{9} \mathrm{C}\left(3 / 2^{-}\right),{ }^{11} \mathrm{C}\left(3 / 2^{-}\right), d\left(1^{+}\right)$, as well as some elementary particles, for example, tau-lepton $\tau(1)$.

The differential cross section for elastic scattering of two particles system with spin structure $1+3 / 2$ accounting spin-orbit splitting is:

$$
\begin{array}{r}
\frac{d \sigma(\theta)}{d \Omega}=\frac{1}{6} \frac{d \sigma_{1 / 2}}{d \Omega}+\frac{1}{3} \frac{d \sigma_{3 / 2}}{d \Omega}+\frac{1}{2} \frac{d \sigma_{5 / 2}}{d \Omega}, \quad \text { (2) } \quad \begin{array}{c}
\text { Coulomb scattering amplitude } \\
\text { following form }
\end{array} \\
f_{c}(\theta)=-\left(\frac{\gamma}{2 k \sin ^{2}(\theta / 2)}\right) \exp \left(i \gamma \ln \left[\sin ^{-2}(\theta / 2)\right]+2 i \sigma_{0}\right),
\end{array}
$$

where the indices and related to the doublet, quartet and quintet scattering states corresponding to the total spin channel $S=1 / 2, S=3 / 2$ and $S=5 / 2$. For the partial cross sections 28 amplitudes have been analytically calculated.

Partial cross sections in (2) is the combination of amplitudes $X=A, B, C \ldots$, defined as a following

$$
X=f_{c} \delta_{v v^{\prime}}+\frac{1}{2 i k} \sum_{\ell=m} X_{j} e^{2 i \sigma_{\ell}} P_{\ell}^{m}(\cos \theta) .
$$

Angular functions $Y_{\ell}^{m}(\theta, 0)$ are converted to the Legendre polynomials $P_{\ell}{ }^{m}(\cos \theta)[13], m=v-v^{\prime}$. Within the selection rules $X_{j}$ is the sum of all possible amplitudes $\alpha^{j}$ with appropriate algebraic coefficients $C_{j}$ given in Tables 1-3:

$$
\begin{gathered}
X_{j}=\sum_{j} \alpha^{j} C_{j}, \\
\alpha^{j}=1-U_{s \ell}^{J^{\pi}} .
\end{gathered}
$$

Coulomb scattering amplitude is rewritten in the where $\gamma$-Coulomb parameter; $k$ - the wave number of the relative particle motion, $k^{2}=2 \mu E / \hbar^{2}$. Coulomb parameter is represented as:

$$
\gamma=\frac{\mu Z_{1} Z_{2} e^{2}}{k \hbar^{2}},
$$

where $Z$-particle charges in units $e, \mu$-reduced mass $E$ - energy of scattering particles in center mass.
The differential cross section of elastic scattering in the doublet state $(S=1 / 2)$ is well known $[3,9,10]$ :

$$
\frac{d \sigma_{1 / 2}}{d \Omega}=\left(\left|A_{1 / 2}\right|^{2}+\left|B_{1 / 2}\right|^{2}\right)
$$

The corresponding coefficients are given in Table 1 . There and after values $m$ complete the construction of (3). Thus $m=0$ for amplitude $A$ and $m=1$ for $B$ in (8). 
Table 1 - doublet spin channel $C_{j}$ coefficients

\begin{tabular}{|c|c|c|}
\hline \multirow{2}{*}{$X$} & \multicolumn{2}{|c|}{$j$} \\
\cline { 2 - 3 } & $\ell+1 / 2$ & $\ell-1 / 2$ \\
\hline$A_{1 / 2}$ & $\ell+1$ & $\ell$ \\
\hline$B_{1 / 2}$ & $-\sqrt{\ell(\ell+1)}$ & $\sqrt{\ell(\ell+1)}$ \\
\hline
\end{tabular}

The differential cross section of elastic scattering in the quartet state $(S=3 / 2)$ is:

$$
\frac{d \sigma_{3 / 2}}{d \Omega}=\frac{1}{2}\left(\left|A_{3 / 2}\right|^{2}+\left|B_{3 / 2}\right|^{2}+\left|C_{3 / 2}\right|^{2}+\left|D_{3 / 2}\right|^{2}+\left|E_{3 / 2}\right|^{2}+\left|F_{3 / 2}\right|^{2}+\left|G_{3 / 2}\right|^{2}+\left|H_{3 / 2}\right|^{2}\right) .
$$

Here amplitudes are calculated by formula (3) $\quad m=0$ for amplitudes $A$ and $B ; m=1$ for $C, D$ and using Table 2 and complementary information: $E ; m=2$ for $F$ and $G ; m=3$ for $H$.

Table 2 - quartet spin channel $C_{j}$ coefficients

\begin{tabular}{|c|c|c|c|c|}
\hline \multirow{2}{*}{$X$} & \multicolumn{4}{|c|}{$J$} \\
\hline & $\ell+3 / 2$ & $\ell+1 / 2$ & $\ell-1 / 2$ & $\ell-3 / 2$ \\
\hline \multirow{2}{*}{$A_{3 / 2}$} & $6(\ell+1)(\ell+2)$ & $2 \ell(\ell+1)$ & $2 \ell(\ell+1)$ & $6 \ell(\ell-1)$ \\
\hline & $2 \ell+3$ & $2 \ell+3$ & $2 \ell-1$ & $2 \ell-1$ \\
\hline \multirow{2}{*}{$B_{3 / 2}$} & $2(\ell+2)(\ell+3)$ & $6(\ell+1)(\ell+2)$ & $6 \ell(\ell-1)$ & $2(\ell-1)(\ell-2)$ \\
\hline & $2 \ell+3$ & $2 \ell+3$ & $2 \ell-1$ & $2 \ell-1$ \\
\hline \multirow{2}{*}{$C_{3 / 2}$} & $6(\ell+2)$ & $2(\ell+3)$ & $2(\ell-2)$ & $6(\ell-1)$ \\
\hline & $2 \ell+3$ & $2 \ell+3$ & $2 \ell-1$ & $2 \ell-1$ \\
\hline \multirow{2}{*}{$D_{3 / 2}$} & $2 \sqrt{3}(\ell+2)$ & $2 \sqrt{3}(\ell+1)$ & $2 \sqrt{3} \ell$ & $2 \sqrt{3}(\ell-1)$ \\
\hline & $2 \ell+3$ & $2 \ell+3$ & $\overline{2 \ell-1}$ & $2 \ell-1$ \\
\hline \multirow{2}{*}{$E_{3 / 2}$} & $2 \sqrt{3}(\ell+2)(\ell+3)$ & $2 \sqrt{3}(\ell+2)(\ell-3)$ & $2 \sqrt{3}(\ell+4)(\ell-1)$ & $2 \sqrt{3}(\ell-1)(\ell-2)$ \\
\hline & $(\ell+1)(2 \ell+3)$ & $\ell(2 \ell+3)$ & $(\ell+1)(2 \ell-1)$ & $\ell(2 \ell-1)$ \\
\hline \multirow{2}{*}{$F_{3 / 2}$} & $2 \sqrt{3}$ & $2 \sqrt{3}$ & $2 \sqrt{3}$ & $2 \sqrt{3}$ \\
\hline & $2 \ell+3$ & $2 \ell+3$ & $2 \ell-1$ & $2 \ell-1$ \\
\hline \multirow{2}{*}{$G_{3 / 2}$} & $2 \sqrt{3}(\ell+3)$ & $2 \sqrt{3}(\ell+6)$ & $2 \sqrt{3}(\ell-5)$ & $2 \sqrt{3}(\ell-2)$ \\
\hline & $\overline{(\ell+1)(2 \ell+3)}$ & $\ell(2 \ell+3)$ & $\overline{(\ell+1)(2 \ell-1)}$ & $\ell(2 \ell-1)$ \\
\hline \multirow{2}{*}{$H_{3 / 2}$} & 2 & 6 & 6 & 2 \\
\hline & $(\ell+1)(2 \ell+3)$ & $\ell(2 \ell+3)$ & $(\ell+1)(2 \ell-1)$ & $\ell(2 \ell-1)$ \\
\hline
\end{tabular}

A similar way, using the Table 3 can be calculated The partial cross-section of the channel is a combination the amplitudes for the quintet spin channel $S=5 / 2$. of 18 independent amplitudes.

$$
\begin{aligned}
& \frac{d \sigma_{5 / 2}}{d \Omega}=\frac{1}{2}\left(\left|A_{5 / 2}\right|^{2}+\left|B_{5 / 2}\right|^{2}+\left|C_{5 / 2}\right|^{2}+\left|D_{5 / 2}\right|^{2}+\left|E_{5 / 2}\right|^{2}+\left|F_{5 / 2}\right|^{2}+\left|G_{5 / 2}\right|^{2}+\left|H_{5 / 2}\right|^{2}+\left|I_{5 / 2}\right|^{2}+\right. \\
& \left.\quad+\left|J_{5 / 2}\right|^{2}+\left|K_{5 / 2}\right|^{2}+\left|L_{5 / 2}\right|^{2}+\left|M_{5 / 2}\right|^{2}+\left|N_{5 / 2}\right|^{2}+\left|O_{5 / 2}\right|^{2}+\left|P_{5 / 2}\right|^{2}+\left|Q_{5 / 2}\right|^{2}+\left|R_{5 / 2}\right|^{2}\right)
\end{aligned}
$$


Table 3 - quintet spin channel $C_{j}$ coefficients

\begin{tabular}{|c|c|c|c|}
\hline \multirow{2}{*}{$X$} & \multicolumn{3}{|c|}{$j$} \\
\hline & $\ell+5 / 2$ & $\ell+3 / 2$ & $\ell+1 / 2$ \\
\hline \multirow{2}{*}{$A_{5 / 2}$} & $10(\ell+1)(\ell+2)(\ell+3)$ & $2(\ell+1)(\ell+2)$ & $4 \ell(\ell+1)(\ell+2)$ \\
\hline & $(2 \ell+3)(2 \ell+5)$ & $(2 \ell+3)(2 \ell+5)$ & $(2 \ell+3)(2 \ell-1)$ \\
\hline \multirow{2}{*}{$B_{5 / 2}$} & $20(\ell+1)(\ell+3)(\ell+4)$ & $36 \ell(\ell+2)(\ell+3)$ & $8(\ell+1)(\ell-3)^{2}$ \\
\hline & $(2 \ell+3)(2 \ell+5)$ & $\overline{(2 \ell+3)(2 \ell+5)}$ & $\overline{(2 \ell+3)(2 \ell-1)}$ \\
\hline \multirow{2}{*}{$C_{5 / 2}$} & $4(\ell+3)(\ell+4)(\ell+5)$ & $20(\ell+2)(\ell+3)(\ell+4)$ & $40(\ell+3)(\ell+1)(\ell-1)$ \\
\hline & $(2 \ell+3)(2 \ell+5)$ & $(2 \ell+3)(2 \ell+5)$ & $(2 \ell+3)(2 \ell-1)$ \\
\hline \multirow{2}{*}{$D_{5 / 2}$} & $10(\ell+2)(\ell+3)$ & $2(\ell+2)(\ell+5)$ & $4(\ell+2)(\ell-2)$ \\
\hline & $(2 \ell+3)(2 \ell+5)$ & $(\ell+3)(2 \ell+5)$ & $\overline{(2 \ell+3)(2 \ell-1)}$ \\
\hline \multirow{2}{*}{$E_{5 / 2}$} & $20 \sqrt{2}(\ell+2)(\ell+3)$ & $4 \sqrt{2}(\ell+2)(3 \ell+5)$ & $8 \sqrt{2}(\ell+1)(\ell+2)$ \\
\hline & $(2 \ell+3)(2 \ell+5)$ & $(2 \ell+3)(2 \ell+5)$ & $(2 \ell+3)(2 \ell-1)$ \\
\hline \multirow{2}{*}{$F_{5 / 2}$} & $20 \sqrt{2}(\ell+3)(\ell+4)$ & $12 \sqrt{2}(\ell+2)(\ell+3)(\ell-5)$ & $8 \sqrt{2}\left(\ell^{2}+4 \ell-6\right)(\ell-3)$ \\
\hline & $(2 \ell+3)(2 \ell+5)$ & $(\ell+1)(2 \ell+3)(2 \ell+5)$ & $\ell(2 \ell+3)(2 \ell-1)$ \\
\hline \multirow{2}{*}{$G_{5 / 2}$} & $4 \sqrt{5}(\ell+3)(\ell+4)$ & $12 \sqrt{5}(\ell+2)(\ell+3)$ & $8 \sqrt{5}(\ell+1)(\ell-3)$ \\
\hline & $(2 \ell+3)(2 \ell+5)$ & $(2 \ell+3)(2 \ell+5)$ & $(2 \ell+3)(2 \ell-1)$ \\
\hline \multirow{2}{*}{$H_{5 / 2}$} & $4 \sqrt{5}(\ell+3)(\ell+4)(\ell+5)$ & $4 \sqrt{5}(\ell+2)(\ell+3)(\ell+4)(3 \ell-5)$ & $8 \sqrt{5}(\ell+3)(\ell-1)(\ell-8)$ \\
\hline & $(\ell+1)(2 \ell+3)(2 \ell+5)$ & $\ell(\ell+1)(2 \ell+3)(2 \ell+5)$ & $\ell(2 \ell+3)(2 \ell-1)$ \\
\hline \multirow{2}{*}{$I_{5 / 2}$} & $20 \sqrt{2}(\ell+3)$ & $4 \sqrt{2}(3 \ell+10)$ & $8 \sqrt{2}(\ell+7)$ \\
\hline & $\overline{(2 \ell+3)(2 \ell+5)}$ & $\overline{(2 \ell+3)(2 \ell+5)}$ & $(2 \ell+3)(2 \ell-1)$ \\
\hline \multirow{2}{*}{$J_{5 / 2}$} & $4 \sqrt{10}(\ell+3)$ & $4 \sqrt{10}(\ell+2)$ & $8 \sqrt{10}(\ell+1)$ \\
\hline & $\overline{(2 \ell+3)(2 \ell+5)}$ & $\overline{(2 \ell+3)(2 \ell+5)}$ & $\overline{(2 \ell+3)(2 \ell-1)}$ \\
\hline \multirow{2}{*}{$K_{5 / 2}$} & $20 \sqrt{2}(\ell+3)(\ell+4)$ & $12 \sqrt{2}(\ell+3)(\ell+10)$ & $8 \sqrt{2}(\ell-3)\left(\ell^{2}-2 \ell-18\right)$ \\
\hline & $\overline{(\ell+2)(2 \ell+3)(2 \ell+5)}$ & $\overline{(\ell+1)(2 \ell+3)(2 \ell+5)}$ & $\ell(\ell+2)(2 \ell+3)(2 \ell-1)$ \\
\hline \multirow{2}{*}{$L_{5 / 2}$} & $4 \sqrt{10}(\ell+3)(\ell+4)(\ell+5)$ & $4 \sqrt{10}(\ell+3)(\ell+4)(\ell-10)$ & $8 \sqrt{10}(\ell+3)\left(\ell^{2}+6 \ell-22\right)$ \\
\hline & $\overline{(\ell+1)(\ell+2)(2 \ell+3)(2 \ell+5)}$ & $\ell(\ell+1)(2 \ell+3)(2 \ell+5)$ & $\ell(\ell+2)(2 \ell+3)(2 \ell-1)$ \\
\hline \multirow{2}{*}{$M_{5 / 2}$} & $4 \sqrt{10}$ & $4 \sqrt{10}$ & $8 \sqrt{10}$ \\
\hline & $\overline{(2 \ell+3)(2 \ell+5)}$ & $\overline{(2 \ell+3)(2 \ell+5)}$ & $\overline{(2 \ell+3)(2 \ell-1)}$ \\
\hline \multirow{2}{*}{$N_{5 / 2}$} & $20(\ell+4)$ & $36(\ell+5)$ & $8(\ell+12)(\ell-3)$ \\
\hline & $(\ell+2)(2 \ell+3)(2 \ell+5)$ & $(\ell+1)(2 \ell+3)(2 \ell+5)$ & $\ell(\ell+2)(2 \ell+3)(2 \ell-1)$ \\
\hline \multirow{2}{*}{$O_{5 / 2}$} & $4 \sqrt{10}(\ell+4)(\ell+5)$ & $4 \sqrt{10}(\ell+15)(\ell+4)$ & $8 \sqrt{10}\left(\ell^{2}-4 \ell-42\right)$ \\
\hline & $(\ell+1)(\ell+2)(2 \ell+3)(2 \ell+5)$ & $\ell(\ell+1)(2 \ell+3)(2 \ell+5)$ & $\ell(\ell+2)(2 \ell+3)(2 \ell-1)$ \\
\hline \multirow{2}{*}{$P_{5 / 2}$} & 20 & 60 & $40(\ell-3)$ \\
\hline & $(\ell+2)(2 \ell+3)(2 \ell+5)$ & $(\ell+1)(2 \ell+3)(2 \ell+5)$ & $\overline{\ell(\ell+2)(2 \ell+3)(2 \ell-1)}$ \\
\hline \multirow{2}{*}{$Q_{5 / 2}$} & $20(\ell+5)$ & $20(3 \ell+20)$ & $40(\ell+17)$ \\
\hline & $\overline{(\ell+1)(\ell+2)(2 \ell+3)(2 \ell+5)}$ & $\overline{\ell(\ell+1)(2 \ell+3)(2 \ell+5)}$ & $\overline{\ell(\ell+2)(2 \ell+3)(2 \ell-1)}$ \\
\hline
\end{tabular}




\begin{tabular}{|c|c|c|c|}
\hline$R_{5 / 2}$ & $-\sqrt{\frac{(\ell-5) !}{(\ell+5) !}}$ & $20 \sqrt{\frac{(\ell-5) !}{(\ell+5) !}}$ & $-10 \sqrt{\frac{(\ell-5) !}{(\ell+5) !}}$ \\
\hline \multirow{2}{*}{$X$} & \multicolumn{3}{|c|}{$j$} \\
\hline & $\ell-1 / 2$ & $\ell-3 / 2$ & $\ell-5 / 2$ \\
\hline \multirow{2}{*}{$A_{5 / 2}$} & $4 \ell(\ell+1)(\ell-1)$ & $2 \ell(\ell+1)(\ell-1)$ & $10 \ell(\ell-1)(\ell-2)$ \\
\hline & $\overline{(2 \ell+3)(2 \ell-1)}$ & $\overline{(2 \ell-1)(2 \ell-3)}$ & $\overline{(2 \ell-1)(2 \ell-3)}$ \\
\hline \multirow{2}{*}{$B_{5 / 2}$} & $8 \ell(\ell+4)^{2}$ & $36(\ell+1)(\ell-1)(\ell-2)$ & $20 \ell(\ell-2)(\ell-3)$ \\
\hline & $\overline{(2 \ell+3)(2 \ell-1)}$ & $(2 \ell-1)(2 \ell-3)$ & $(2 \ell-1)(2 \ell-3)$ \\
\hline \multirow{2}{*}{$C_{5 / 2}$} & $40 \ell(\ell+2)(\ell-2)$ & $20(\ell-1)(\ell-2)(\ell-3)$ & $4(\ell-2)(\ell-3)(\ell-4)$ \\
\hline & $(2 \ell+3)(2 \ell-1)$ & $(2 \ell-1)(2 \ell-3)$ & $(2 \ell-1)(2 \ell-3)$ \\
\hline \multirow{2}{*}{$D_{5 / 2}$} & $4(\ell+3)(\ell-1)$ & $2(\ell-1)(\ell-4)$ & $10(\ell-1)(\ell-2)$ \\
\hline & $\overline{(2 \ell+3)(2 \ell-1)}$ & $\overline{(2 \ell-1)(2 \ell-3)}$ & $\overline{(2 \ell-1)(2 \ell-3)}$ \\
\hline \multirow{2}{*}{$E_{5 / 2}$} & $8 \sqrt{2} \ell(\ell-1)$ & $4 \sqrt{2}(\ell-1)(3 \ell-2)$ & $20 \sqrt{2}(\ell-1)(\ell-2)$ \\
\hline & $\overline{(2 \ell+3)(2 \ell-1)}$ & $(2 \ell-1)(2 \ell-3)$ & $(2 \ell-1)(2 \ell-3)$ \\
\hline \multirow{2}{*}{$F_{5 / 2}$} & $8 \sqrt{2}(\ell+4)\left(\ell^{2}-2 \ell-9\right)$ & $12 \sqrt{2}(\ell+6)(\ell-1)(\ell-2)$ & $20 \sqrt{2}(\ell-2)(\ell-3)$ \\
\hline & $(\ell+1)(2 \ell+3)(2 \ell-1)$ & $\ell(2 \ell-1)(2 \ell-3)$ & $(2 \ell-1)(2 \ell-3)$ \\
\hline \multirow{2}{*}{$G_{5 / 2}$} & $8 \sqrt{5} \ell(\ell+4)$ & $12 \sqrt{5}(\ell-1)(\ell-2)$ & $4 \sqrt{5}(\ell-2)(\ell-3)$ \\
\hline & $\overline{(2 \ell+3)(2 \ell-1)}$ & $\overline{(2 \ell-1)(2 \ell-3)}$ & $\overline{(2 \ell-1)(2 \ell-3)}$ \\
\hline \multirow{2}{*}{$H_{5 / 2}$} & $8 \sqrt{5}(\ell+9)(\ell+2)(\ell-2)$ & $4 \sqrt{5}(3 \ell+8)(\ell-1)(\ell-2)(\ell-3)$ & $4 \sqrt{5}(\ell-2)(\ell-3)(\ell-4)$ \\
\hline & $(\ell+1)(2 \ell+3)(2 \ell-1)$ & $\ell(\ell+1)(2 \ell-1)(2 \ell-3)$ & $\ell(2 \ell-1)(2 \ell-3)$ \\
\hline \multirow{2}{*}{$I_{5 / 2}$} & $8 \sqrt{2}(\ell-6)$ & $4 \sqrt{2}(3 \ell-7)$ & $20 \sqrt{2}(\ell-2)$ \\
\hline & $\overline{(2 \ell+3)(2 \ell-1)}$ & $\overline{(2 \ell-1)(2 \ell-3)}$ & $\overline{(2 \ell-1)(2 \ell-3)}$ \\
\hline \multirow{2}{*}{$J_{5 / 2}$} & $8 \sqrt{10} \ell$ & $4 \sqrt{10}(\ell-1)$ & $4 \sqrt{10}(\ell-2)$ \\
\hline & $\overline{(2 \ell+3)(2 \ell-1)}$ & $\overline{(2 \ell-1)(2 \ell-3)}$ & $\overline{(2 \ell-1)(2 \ell-3)}$ \\
\hline \multirow{2}{*}{$K_{5 / 2}$} & $8 \sqrt{2}(\ell+4)\left(\ell^{2}+4 \ell-15\right)$ & $12 \sqrt{2}(\ell-2)(\ell-9)$ & $20 \sqrt{2}(\ell-2)(\ell-3)$ \\
\hline & $\overline{(\ell+1)(\ell-1)(2 \ell+3)(2 \ell-1)}$ & $\overline{\ell(2 \ell-1)(2 \ell-3)}$ & $\overline{(\ell-1)(2 \ell-1)(2 \ell-3)}$ \\
\hline \multirow{2}{*}{$L_{5 / 2}$} & $8 \sqrt{10}(\ell-2)\left(\ell^{2}-4 \ell-27\right)$ & $4 \sqrt{10}(\ell-2)(\ell-3)(\ell+11)$ & $4 \sqrt{10}(\ell-2)(\ell-3)(\ell-4)$ \\
\hline & $\overline{(\ell+1)(\ell-1)(2 \ell+3)(2 \ell-1)}$ & $\ell(\ell+1)(2 \ell-1)(2 \ell-3)$ & $\ell(\ell-1)(2 \ell-1)(2 \ell-3)$ \\
\hline \multirow{2}{*}{$M_{5 / 2}$} & $8 \sqrt{10}$ & $4 \sqrt{10}$ & $4 \sqrt{10}$ \\
\hline & $\overline{(2 \ell+3)(2 \ell-1)}$ & $\overline{(2 \ell-1)(2 \ell-3)}$ & $\overline{(2 \ell-1)(2 \ell-3)}$ \\
\hline \multirow{2}{*}{$N_{5 / 2}$} & $8(\ell+4)(\ell-11)$ & $36(\ell-4)$ & $20(\ell-3)$ \\
\hline & $\overline{(\ell+1)(\ell-1)(2 \ell+3)(2 \ell-1)}$ & $\overline{\ell(2 \ell-1)(2 \ell-3)}$ & $\overline{(\ell-1)(2 \ell-1)(2 \ell-3)}$ \\
\hline \multirow{2}{*}{$O_{5 / 2}$} & $8 \sqrt{10}\left(\ell^{2}+6 \ell-37\right)$ & $4 \sqrt{10}(\ell-3)(\ell-14)$ & $4 \sqrt{10}(\ell-3)(\ell-4)$ \\
\hline & $\overline{(\ell+1)(\ell-1)(2 \ell+3)(2 \ell-1)}$ & $\ell(\ell+1)(2 \ell-1)(2 \ell-3)$ & $\overline{\ell(\ell-1)(2 \ell-1)(2 \ell-3)}$ \\
\hline \multirow{2}{*}{$P_{5 / 2}$} & $40(\ell+4)$ & 60 & 20 \\
\hline & $\overline{(\ell+1)(\ell-1)(2 \ell+3)(2 \ell-1)}$ & $\overline{\ell(2 \ell-1)(2 \ell-3)}$ & $\overline{(\ell-1)(2 \ell-1)(2 \ell-3)}$ \\
\hline \multirow{2}{*}{$Q_{5 / 2}$} & $40(\ell-16)$ & $20(3 \ell-17)$ & $20(\ell-4)$ \\
\hline & $\overline{(\ell+1)(\ell-1)(2 \ell+3)(2 \ell-1)}$ & $\ell(\ell+1)(2 \ell-1)(2 \ell-3)$ & $\ell(\ell-1)(2 \ell-1)(2 \ell-3)$ \\
\hline \multirow[t]{2}{*}{$R_{5 / 2}$} & $10 \sqrt{\frac{(\ell-5) !}{(\ell-2) !}}$ & $-5 \sqrt{\frac{(\ell-5) !}{(0) 5) !}}$ & $\sqrt{\frac{(\ell-5) !}{(\ell)-5)}}$ \\
\hline & $\sqrt{(\ell+5) !}$ & $\sqrt{(\ell+5) !}$ & $\sqrt{(\ell+5) !}$ \\
\hline
\end{tabular}


Here $m=0$ for amplitudes $A, B$ and $C ; m=1$ for $D, E, F, G$ and $H ; m=2$ for $I, J, K$ and $L ; m=3$ for $M, N$ and $O ; m=4$ f or $P$ и $Q, m=5$ for $R$.

\section{Conclusion}

Present work for the first time reported the formulas for the calculation of differential cross sections in scattering channels with spins $3 / 2$ and $5 / 2$ in addition to $1 / 2$. The obtained representations are universal and can be used for a wide range of problems in nuclear and particle physics. Namely their different combinations depending on the spins of scattering particles cover cases $s_{1}+s_{2}=1+1 / 2,1+3 / 2,2+1 / 2$. Due to the fact that the orbital quantum number can take any value in (3), these formulas are applicable for the calculation of reactions proceeding at low and ultra - low astrophysical energies, as well as for the high-energy processes.

\section{References}

1 Ishhanov B.S., Kapitonov I.M., Judin N.P. Chasticy i atomnye jadra.- M.: Izdatel'stvo LKI. 2007. - P. 584.

2 Kapitonov I.M., Ishhanov B.S., Tutyn' I.A. Nukleosintez vo Vselennoj. - M.: Librokom. 2009. - P. 202.

3 Lane A.M., Thomas R.G. R-Matrix Theory of Nuclear Reactions // Reviews of Modern Physics. - 1958. - Vol. 30. - P. 257-353.
4 Ohlsen G.G. Polarization transfer and spin correlation experiments in nuclear physics // Rep. Prog. Phys. - 1972. - Vol 35. - P. 717-801.

5 Brown L., Stainer E., Arnold L.G. and Seyler R.G. Polarization and phase shifts in ${ }^{7} \mathrm{Li}(\mathrm{p}, \mathrm{p})^{7} \mathrm{Li}$ from 0.4 to $0.25 \mathrm{MeV}$ and the structure of ${ }^{8}$ Be // Nucl. Phys. - 1973. - Vol. A206. - P. 353373.

6 Seyler R.G. Polarization from scattering polarized spin-1/2 on unpolarized spin-1 particles // Nucl. Phys. - 1969. - Vol. A124. - P. 253-272.

7 Nemec O.F., Jasnogorodskij A.M. Poljarizacionnye issledovanija $\mathrm{v}$ jadernoj fizike. Kiev: Naukova Dumka. - 1980. - P. 347.

8 Kolybasov V.M. Obshhie svojstva matricy rassejanija i poljarizacionnye jeffekty $\mathrm{v}$ prjamyh reakcijah. - M.: MIFI. - 1971. - P. 129.

9 Davydov A.S. Teorija atomnogo jadra. M.: Fizmatgiz. - 1958. - P. 612.

10 Sitenko A.G. Teorija jadernyh reakcij. - M: Jenergoatomizdat. - 1983. - P. 352.

11 Dubovichenko S.B. Svojstva legkih atomnyh jader $\mathrm{v}$ potencial'noj klasternoj modeli. Almaty: Daneker. - 2004. - P. 247.

12 Dubovichenko S.B. Termojadernye processy Vselennoj. Tom 7. - Almaty: Izd. Astrofizicheskogo instituta im. V.G. Fesenkova "NCKIT" NKA RK. - 2011. - P. 402.

13 Varshalovich D.A., Moskalev A.N., Hersonskij V.K. Kvantovaja teorija uglovogo momenta. - L.: Nauka. - 1975. - P. 439. 